\title{
Accessibility, longitudinality, comprehensiveness and coordination of care: our proposal
}

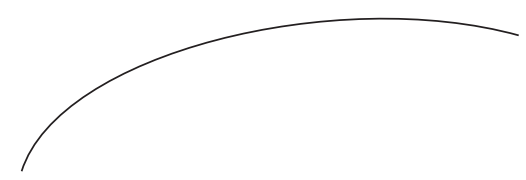

We begin the year by announcing a significant achievement. From this edition onwards, Revista Brasileira de Geriatria e Gerontologia (the Brazilian Journal of Geriatrics and Gerontology) (RBGG) will be published bimonthly. We are establishing ourselves as one of the most relevant publications in the field of human aging, from the perspective of public health. And all this without forgetting our role as a fosterer of challenging and contemporary scientific proposals, based around increasing the quality of life of the elderly.

With the boldness that has always been our trademark, we could not begin this new phase without a reflection for the professionals who work in our field. We will begin with a diagnosis: healthcare management needs to change. This is one of the biggest challenges faced by the sector today.

A growing number of scientific and academic studies in the field of health have addressed this theme, in the search for models and alternatives that meet our needs more effectively. However, one of the difficulties with such a transformation is that a large number of health professionals who work exclusively in the area of care seem unaware that change is fundamental for the sustainability of the health care model. The predominant system is still the "Flexnerian Biomedical Model", which focuses on a reductionist view of health, based on individual care and a curative approach. Remarkably, this model dates from 1920!

While many doctors talk about offering a more contemporary focus based on monitoring, the anticipation of illness and preventative actions, the day to day reality of their work is rather different. The relationship between words and deeds is utterly schizophrenic. These health professionals, who hold considerable influence in society, collaborate so that nothing changes and the hospital-centered logic remains. However, perhaps due to economic factors, the mood of change seems to be growing, and this group, while still prevailing, is losing space. The challenge, therefore, is how to change the care model if many professionals insist on maintaining outdated practices?

There are a variety of healthcare models around the world. Some have become hegemonic at a given historical moment, usually in response to the limitations of the previously dominant system. Over time, their own frailties make them the target of criticism and lead to their replacement. 
Factors such as population aging, the prevalence of chronic and degenerative diseases, obesity and the incorporation of new technologies are exerting great pressure on public and private health systems. It is essential, therefore, to develop solutions that combine quality and sustainability.

It is time that we understood that continuing to work as we have been doing will produce the same results - but at higher costs. We need to ensure the centrality of the patient and his or her requirements, which implies a new relationship between the client and the services he or she receives, ensuring coordinated, integrated care, rather than excessive fragmentation. It is therefore necessary that all information is shared access the healthcare team, which should include a referral health professional, with training based on care and monitoring. This strategy aims to ensure a better outcome for the user, his or her family and the service provider. It is also focused on economic viability through the elimination of waste.

In short, what is sought is a management model based on the principles of Integral Health Care which - with great skill but at the same time, simplicity - will be resolutive and sustainable. We believe that it is possible to change, to do things differently, offering users better quality, but no more expensive, health care. On the contrary, we are describing a qualified healthcare system, which is capable of reducing waste through the proper use of resources, of monitoring and anticipating, of engaging with and creating loyalty among clients, of involving families and of producing the results for which we have waited for so long. At UnATI, our institution, and from other Brazilian and international examples, there is evidence that it is possible to practice quality medicine at a lower cost. 